\title{
Differential Equations of Motion for Naturally Curved and Twisted Composite Space Beams
}

\author{
Ying Hao (D), Wei He, and Yanke Shi \\ School of Civil Engineering and Communication, North China University of Water Resources and Electric Power, \\ Zhengzhou 450045, China
}

Correspondence should be addressed to Ying Hao; yinghao_ncwu@163.com

Received 24 August 2017; Revised 30 March 2018; Accepted 16 April 2018; Published 15 May 2018

Academic Editor: Toshiaki Natsuki

Copyright (C) 2018 Ying Hao et al. This is an open access article distributed under the Creative Commons Attribution License, which permits unrestricted use, distribution, and reproduction in any medium, provided the original work is properly cited.

The differential equations of motion for naturally curved and twisted elastic space beams made of anisotropic materials with noncircular cross sections, being a coupled system consisting of 14 second-order partial differential equations with variable coefficients, are derived theoretically. The warping deformation of beam's cross section, as a new design factor, is incorporated into the differential equations in addition to the anisotropy of material, the curvatures of the rod axis, the initial twist of the cross section, the rotary inertia, and the shear and axial deformations. Numerical examples show that the effect of warping deformation on the natural frequencies of the beam is significant under certain geometric and boundary conditions. This study focuses on improving and consummating the traditional theories to build a general curve beam theory, thereby providing new scientific research reference and design principle for curve beam designers.

\section{Introduction}

Composite space beams with noncircular cross sections have extensive applications in civil, mechanical, aircraft, and aerospace constructions given their outstanding engineering properties, such as specific strength, specific stiffness, weight, and fatigue life over metallic counterparts. The static and dynamic characteristics of composite beams have attracted considerable attention in recent decades.

Elastic couplings occur among all the forms of deformation given the anisotropy of the material. Moreover, in-plane and out-of-plane displacements may be coupled. Therefore, the differential equations of motion, obtained by the $3 \mathrm{D}$ elastic theory under isothermal conditions, become more complex by allowing initial curvature and torsion. EulerBernoulli and Timoshenko beam theories are unable to accurately describe the behavior of composite beams.

Many researchers have conducted profound and careful research on nonclassical influences, such as transverse shear deformations, torsion-related warping, and several elastic couplings that can develop into composite beams. The natural frequencies and modes of composite beams under various boundary conditions are obtained by using the first-order shear deformation theory [1]. Based on the third-order shear theory, the forced vibration response of composite beams is investigated [2]. Li et al. [3] discussed the influence of different support conditions on the natural frequency of composite beams using many numerical examples. In this study, the effect of shear deformation is noted. Ramalingeswara and Ganesan [4] and Zapfe and Lesieutre [5] delivered the natural frequencies of laminated beams by using higher order shear theory and iterative method.

Most of abovementioned studies [1-5] focus on straight beams. The research on the dynamic behavior is far less in composite curved beams than in straight beams considering the curvature and torsion. Yildirim [6] studied the free vibration of symmetrically orthogonal laminated curved beams, in which rotary inertia and axial and shear deformation effects are considered. Tseng et al. [7] studied the free vibration of laminated curved beams with variable curvature based on Timoshenko theory. However, a transverse shear strain is constant along the thickness direction, and the shear shape factor was used in this research to adjust the lateral shear stiffness. The accuracy of the results significantly depends on a selected shear shape factor. Matsunaga $[8,9]$ used 1D high-order theory to solve the natural frequencies and 


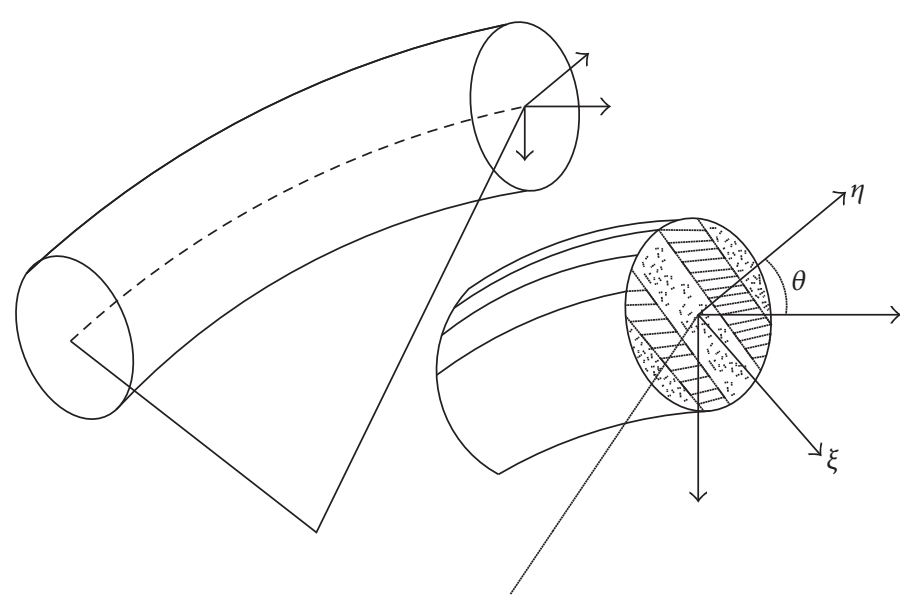

FIGURE 1: Geometry of a naturally curved and twisted anisotropic beam.

buckling loads of composite curved beams subjected to axial concentrated force considering the shear deformation and moment of inertia. Baba and Thoppul [10] explored the flexural strength and vibration characteristics of sandwich laminated curved beams determined the effects of laminated debonding and curvature on their natural frequencies. Yildirim [11] derived the differential equations of motion of spatial curved bars of anisotropic materials, but the examples in this research remain straight beams.

The abovementioned studies [1-11] included one or several items of the anisotropy of the rod material, the curvature and torsion of the rod axis, the effects of rotary inertia, and the shear and axial deformations. The effect of the warping deformation of the cross section on natural frequencies is disregarded. In recent years, many researchers have attempted to establish generalized curved beam theory with warping effect. Kim et al. [12] developed a finite elementbased beam analysis for anisotropic beams with arbitrary shaped cross sections through asymptotic expansion method. Displacements at each order are split into fundamental and warping solutions in their research. The influence of the warping effect is analyzed through examples, thereby demonstrating the importance of including the warping degrees of freedom in the dynamic analysis [13]. Yu et al. [14] formulated a generalized finite element-based cross-sectional analysis for inhomogeneous, initial curved and twisted, anisotropic beams, in which the transverse shear deformation is classified as part of the warping field. Ghafari and Rezaeepazhand [15] discussed the free vibration of rotating composite beams with an arbitrary cross section on the basis of dimensional reduction method. These authors decomposed a $3 \mathrm{D}$ elasticity problem of the composite beam into a $2 \mathrm{D}$ crosssectional and a $1 \mathrm{D}$ beam analysis. Recently, Sapountzakis and Tsiptsis [16-19] used the Analog Equation Method and the Isogeometric Tool to solve the static and dynamic analysis of the curved beams with arbitrary cross section taking into account nonuniform warping and shear deformation effects. Nonuniform warping distributions are taken into account by employing four independent warping parameters multiplying a shear warping function in each direction and two torsional warping functions in these papers.

As an extension of [11, 20-22], the essential features and novel aspects of the present formulation compared with previous ones are summarized as follows.

(i) The improved beam theory is capable of spatial curved beams with arbitrary cross section, variable curvature, and composite material considering warping effect.

(ii) The warping function of unidirectional composite beam with rectangular cross section can be obtained using the separation of variables method discussed in [23].

(iii) Numerical examples are presented for two 3D curved beams with constant curvature and torsion. The results show that the effect of the warping deformation on natural frequencies of the beam is significant under certain geometric and boundary conditions.

(iv) The influence of the warping deformation on the natural frequency is enhanced with the increase in the anisotropy of materials.

\section{Geometrical Characteristics of a Space Anisotropic Beam}

Considering a naturally curved and twisted anisotropic beam in space in Figure 1, t, $\mathbf{n}, \mathbf{b}$ indicate the tangential, normal, and binormal unit vectors of the beam axis, respectively. A second rectangular Cartesian frame $(s, \xi, \eta)$ is introduced to consider the initial twist of the cross section. The angle between the $\xi$ - and $n$-axes is $\theta(s)$. For a smooth curve, the Frenet-Serret formulae are

$$
\begin{aligned}
\mathbf{t}^{\prime} & =k_{1} \mathbf{n}, \\
\mathbf{n}^{\prime} & =-k_{1} \mathbf{t}+k_{2} \mathbf{b}, \\
\mathbf{b}^{\prime} & =-k_{2} \mathbf{n},
\end{aligned}
$$


where ()$^{\prime}=d / d s$ and $s, k_{1}, k_{2}$ are the arc coordinate, the curvature, and torsion of the curve, respectively. In order to take into account the initial twist of the cross section, a new Cartesian reference frame $\left(x_{1}, \xi, \eta\right)$ is introduced such that the $x_{1}$-axis is in the direction of $\mathbf{t}$. If the unit vectors of $O \xi$ and $O \eta$ are represented by $\mathbf{i}_{\xi}$ and $\mathbf{i}_{\eta}$, then

$$
\begin{aligned}
& \mathbf{i}_{\xi}=\mathbf{n} \cos \theta+\mathbf{b} \sin \theta \\
& \mathbf{i}_{\eta}=-\mathbf{n} \sin \theta+\mathbf{b} \cos \theta .
\end{aligned}
$$

With simultaneous equations (1) and (2), we have

$$
\left\{\begin{array}{l}
\mathbf{t}^{\prime} \\
\mathbf{i}_{\xi}^{\prime} \\
\mathbf{i}_{\eta}^{\prime}
\end{array}\right\}=\left[\begin{array}{ccc}
0 & k_{\eta} & -k_{\xi} \\
-k_{\eta} & 0 & k_{s} \\
k_{\xi} & -k_{s} & 0
\end{array}\right]\left\{\begin{array}{c}
\mathbf{t} \\
\mathbf{i}_{\xi} \\
\mathbf{i}_{\eta}
\end{array}\right\},
$$

where $k_{\xi}=k_{1} \sin \theta, k_{\eta}=k_{1} \cos \theta, k_{s}=k_{2}+\theta^{\prime}$.

\section{Constitutive Equations}

The following assumptions are used in the present study: the beam material is linearly elastic, homogeneous, and anisotropic. The pretwisting of the cross section is neglected $\left(k_{\xi}=0\right)$. The generalized Hooke's law for linearly elastic materials is

$$
\boldsymbol{\sigma}_{i}=\mathbf{C}_{i j} \mathbf{e}_{j}
$$

or

$$
\mathbf{e}_{i}=\mathbf{S}_{i j} \boldsymbol{\sigma}_{j} \quad(i, j=1,2, \ldots, 6),
$$

where $\mathbf{C}_{i j}, \mathbf{S}_{i j}$ are the stiffness and flexibility matrices, correspondingly. Let

$$
\begin{aligned}
& \sigma_{1}=\sigma_{s}, \\
& \sigma_{2}=\sigma_{\xi}, \\
& \sigma_{3}=\sigma_{\eta}, \\
& \sigma_{4}=\tau_{\xi \eta}, \\
& \sigma_{5}=\tau_{s \eta}, \\
& \sigma_{6}=\tau_{s \xi}, \\
& e_{1}=e_{s s}, \\
& e_{2}=e_{\xi \xi}, \\
& e_{3}=e_{\eta \eta}, \\
& e_{4}=2 e_{\xi \eta}, \\
& e_{5}=2 e_{s \eta}, \\
& e_{6}=2 e_{s \xi} .
\end{aligned}
$$

The following assumptions on stresses are valid in the classical rod theory.

$$
\sigma_{2}=\sigma_{3}=\sigma_{4}=0 .
$$

$\sigma_{\beta}(\beta=1,5,6)$ can be represented as $e_{\beta}(\beta=1,5,6)$ by solving (7) and (5) simultaneously. Then, the results are substituted to the residual expressions of (5), and the strain components $e_{m}(m=2,3,4)$ are related to $e_{\beta}(\beta=1,5,6)$ by

$$
e_{m}=S_{m n} \alpha_{n \beta} e_{\beta} \quad(m=2,3,4 ; n, \beta=1,5,6) .
$$

In (8),

$$
\begin{aligned}
& \alpha_{11}=\frac{S_{55} S_{66}-S_{56}^{2}}{\Delta}, \\
& \alpha_{55}=\frac{S_{11} S_{66}-S_{16}^{2}}{\Delta}, \\
& \alpha_{66}=\frac{S_{11} S_{55}-S_{15}^{2}}{\Delta}, \\
& \alpha_{15}=\alpha_{51}=\frac{S_{16} S_{56}-S_{15} S_{66}}{\Delta}, \\
& \alpha_{16}=\alpha_{61}=\frac{S_{15} S_{56}-S_{16} S_{55}}{\Delta}, \\
& \alpha_{56}=\alpha_{65}=\frac{S_{15} S_{16}-S_{11} S_{56}}{\Delta} ; \\
& \Delta=\operatorname{det}\left[\begin{array}{lll}
S_{11} & S_{15} & S_{16} \\
S_{15} & S_{55} & S_{56} \\
S_{16} & S_{56} & S_{66}
\end{array}\right] .
\end{aligned}
$$

So, $\sigma_{\beta}(\beta=1,5,6)$ is only related to $e_{\beta}(\beta=1,5,6)$ but unrelated to $e_{m}(m=2,3,4)$ when (8) is substituted to (4). Then, (4) is reduced to

$$
\begin{aligned}
\sigma_{k}=\left(C_{k \beta}+C_{k m} S_{m n} \alpha_{n \beta}\right) e_{\beta} \triangleq Q_{k \beta} e_{\beta} ; \\
(k, \beta, n=1,5,6 ; m=2,3,4) .
\end{aligned}
$$

The displacement components of an arbitrary point of the beam are given as $[20-22,24]$

$$
\begin{aligned}
\mathbf{u}(s, \xi, \eta, t)= & W(s, \xi, \eta, t) \mathbf{t}+U(s, \xi, \eta, t) \mathbf{i}_{\xi} \\
& +V(s, \xi, \eta, t) \mathbf{i}_{\eta} .
\end{aligned}
$$

$\mathbf{u}(s, \xi, \eta, t)$ is not only a function of coordinates $s, \xi, \eta$, but also a function of time under dynamic loads, in which

$$
\begin{aligned}
U(s, \xi, \eta, t)= & u_{\xi}(s, t)-\eta \varphi_{s}(s, t) \\
V(s, \xi, \eta, t)= & u_{\eta}(s, t)+\xi \varphi_{s}(s, t) \\
W(s, \xi, \eta, t)= & u_{s}(s, t)+\eta \varphi_{\xi}(s, t)-\xi \varphi_{\eta}(s, t) \\
& +\alpha(s, t) \varphi(\xi, \eta)
\end{aligned}
$$

where $u_{\xi}(s, t), u_{\eta}(s, t), u_{s}(s, t)$ are the three translations, $\varphi_{\xi}(s, t), \varphi_{\eta}(s, t), \varphi_{s}(s, t)$ are the three rotations, $\alpha(s, t)$ is a generalized warping coordinate which is used to describe the decay of deformation along beam length, and $\varphi(\xi, \eta)$ is the 
warping function of Saint-Venant's torsion. Let $e_{s s}, e_{s \xi}, e_{s \eta}$ be the axial and shear strain components, respectively,

$$
\begin{aligned}
g e_{s s}= & \varepsilon_{s}+\eta \omega_{\xi}-\xi \omega_{\eta}+g \varphi \alpha^{\prime} \\
& +k_{s}\left[\left(\frac{\partial \varphi}{\partial \xi}\right) \eta-\left(\frac{\partial \varphi}{\partial \eta}\right) \xi\right] \alpha \\
2 g e_{s \xi}= & G_{\xi} \varepsilon_{\xi}-\varphi_{\eta}-\eta \omega_{s}+\left[g\left(\frac{\partial \varphi}{\partial \xi}\right)+k_{\eta} \varphi\right] \alpha \\
2 g e_{s \eta}= & G_{\eta} \varepsilon_{\eta}+\varphi_{\xi}+\xi \omega_{s}+\left[g\left(\frac{\partial \varphi}{\partial \eta}\right)+k_{\xi} \varphi\right] \alpha,
\end{aligned}
$$

in which $G_{\xi}, G_{\eta}$ are shear correction factors, and $g=1-\xi k_{\eta}+$ $\eta k_{\xi}$. Based on the assumption that the initial curvature of the beam is gentle, then $g \approx 1$. Let

$$
\begin{aligned}
& \varepsilon_{s}=u_{s}^{\prime}-k_{\eta} u_{\xi}+k_{\xi} u_{\eta}, \\
& \varepsilon_{\xi}=u_{\xi}^{\prime}-k_{s} u_{\eta}+k_{\eta} u_{s}, \\
& \varepsilon_{\eta}=u_{\eta}^{\prime}-k_{\xi} u_{s}+k_{s} u_{\xi}, \\
& \omega_{s}=\varphi_{s}^{\prime}-k_{\eta} \varphi_{\xi}+k_{\xi} \varphi_{\eta}, \\
& \omega_{\xi}=\varphi_{\xi}^{\prime}-k_{s} \varphi_{\eta}+k_{\eta} \varphi_{s}, \\
& \omega_{\eta}=\varphi_{\eta}^{\prime}-k_{\xi} \varphi_{s}+k_{s} \varphi_{\xi} .
\end{aligned}
$$

Let, for convenience, the resultant forces and moments on the cross section be expressed as $Q_{1}=Q_{s}, Q_{5}=Q_{\eta}, Q_{6}=Q_{\xi}$, $M_{1}=M_{s}, M_{5}=M_{\eta}, M_{6}=M_{\xi}$ and shown as

$$
\begin{aligned}
Q_{i} & =\iint_{A} \sigma_{i} d A ; \\
M_{i} & =\varepsilon_{i j k} \iint_{A} x_{j} \sigma_{k} d A ;
\end{aligned}
$$

$$
(i, j, k=1,5,6)
$$

where $\varepsilon_{i j k}$ is the permutation tensor and $x_{1}=s ; x_{5}=\eta ; x_{6}=$ $\xi$.

The generalized warping moment is

$$
T=\iint_{A} \varphi \sigma_{1} d A .
$$

The constitutive equations expressed by six displacement functions $u_{i}(s), \varphi_{i}(s)(i=s, \xi, \eta)$ and the generalized warping coordinate $\alpha(s)$ can be obtained by substituting (10), (13), and (14) into the above formulations (15)-(16).

\section{Equations of Motion}

The external force and moments per unit length of the beam axis are expressed as $\mathbf{q}^{\mathrm{ex}}(s, t)=\left\{q_{s}^{\mathrm{ex}}(s, t), q_{\xi}^{\mathrm{ex}}(s, t), q_{\eta}^{\mathrm{ex}}(s, t)\right\}$, $\mathbf{m}^{\mathrm{ex}}(s, t)=\left\{m_{s}^{\mathrm{ex}}(s, t), m_{\xi}^{\mathrm{ex}}(s, t), m_{\eta}^{\mathrm{ex}}(s, t)\right\}$. Also let $\mathbf{q}^{\mathrm{in}}(s, t)=$ $\left\{q_{s}^{\text {in }}(s, t), q_{\xi}^{\text {in }}(s, t), q_{\eta}^{\text {in }}(s, t)\right\}, \mathbf{m}^{\text {in }}(s, t)=\left\{m_{s}^{\text {in }}(s, t), m_{\xi}^{\text {in }}(s, t)\right.$, $\left.m_{\eta}^{\text {in }}(s, t)\right\}$ be the inertial force and the inertial moment per unit length of the rod axis. Owing to the displacements and the rotations that are assumed to be infinitesimal, the equations of motion can be written as $[11,24]$

$$
\begin{array}{r}
\frac{\partial \mathbf{Q}(s, t)}{\partial s}+\mathbf{q}^{\mathrm{ex}}(s, t)=\mathbf{q}^{\mathrm{in}}(s, t), \\
\frac{\partial \mathbf{M}(s, t)}{\partial s}+\mathbf{t} \times \mathbf{Q}(s, t)+\mathbf{m}^{\mathrm{ex}}(s, t)=\mathbf{m}^{\mathrm{in}}(s, t),
\end{array}
$$

in which

$$
\begin{aligned}
q_{s}^{\text {in }}(s, t)= & \iint_{A} \rho \frac{\partial^{2} u_{s}(s, t)}{\partial t^{2}} d \xi d \eta ; \\
q_{\xi}^{\text {in }}(s, t)= & \iint_{A} \rho \frac{\partial^{2} u_{\xi}(s, t)}{\partial t^{2}} d \xi d \eta ; \\
q_{\eta}^{\text {in }}(s, t)= & \iint_{A} \rho \frac{\partial^{2} u_{\eta}(s, t)}{\partial t^{2}} d \xi d \eta ; \\
m_{s}^{\text {in }}(s, t)= & \iint_{A} \rho \xi \frac{\partial^{2} u_{\eta}(s, t)}{\partial t^{2}} d \xi d \eta \\
& -\iint_{A} \rho \eta \frac{\partial^{2} u_{\xi}(s, t)}{\partial t^{2}} d \xi d \eta ; \\
m_{\xi}^{\text {in }}(s, t)= & \iint_{A} \rho \eta \frac{\partial^{2} u_{s}(s, t)}{\partial t^{2}} d \xi d \eta ; \\
m_{\eta}^{\text {in }}(s, t)= & -\iint_{A} \rho \xi \frac{\partial^{2} u_{s}(s, t)}{\partial t^{2}} d \xi d \eta .
\end{aligned}
$$

The equation of motion in a scalar form can be obtained by substituting (12)-(14) into (17). The equations of $\partial u_{s}(s, t) / \partial s, \partial u_{\xi}(s, t) / \partial s, \partial u_{\eta}(s, t) / \partial s, \partial \varphi_{s}(s, t) / \partial s, \partial \varphi_{\xi}(s, t) / \partial s$, $\partial \varphi_{\eta}(s, t) / \partial s, \partial \alpha(s, t) / \partial s$ can be obtained by solving the abovementioned equations simultaneously. The obtained results are substituted into the derivatives of the generalized warping moment to determine the final differential equations of motion of naturally curved and twisted elastic space beams of anisotropic materials.

$$
\frac{\partial T(s, t)}{\partial s}=\left(\iint \sigma_{s} \varphi d \xi d \eta\right)^{\prime} .
$$

\section{Applications}

5.1. Differential Equations of Motion for the Examples. A simple example is developed here for illustration. Two cylindrical helical springs with rectangular cross section can be regarded as 3D curved beams with constant curvature and torsion (Figure 2). The warping function of unidirectional composite beam with rectangular cross section (Figure 3) can be obtained using the separation of variables method [23]:

$$
\begin{gathered}
\varphi(\xi, \eta)=\xi \eta-\sum_{n=1,3,5}^{\infty}\left(\frac{c_{n} e^{\alpha \gamma_{n} b}}{\alpha \gamma_{n}\left(1+e^{2 \alpha \gamma_{n} b}\right)} e^{\alpha \gamma_{n} \eta}\right. \\
\left.-\frac{c_{n} e^{\alpha \gamma_{n} b}}{\alpha \gamma_{n}\left(1+e^{2 \alpha \gamma_{n} b}\right)} e^{-\alpha \gamma_{n} \eta}\right) \sin \left(\gamma_{n} \xi\right),
\end{gathered}
$$

where $\alpha=\sqrt{G_{x \xi} / G_{x \eta}}, \gamma_{n}=n \pi / 2 a, c_{n}=16 a^{2}(-1)^{(n-1) / 2} / n^{2} \pi^{2}$, $(n=1,3,5 \cdots)$. 


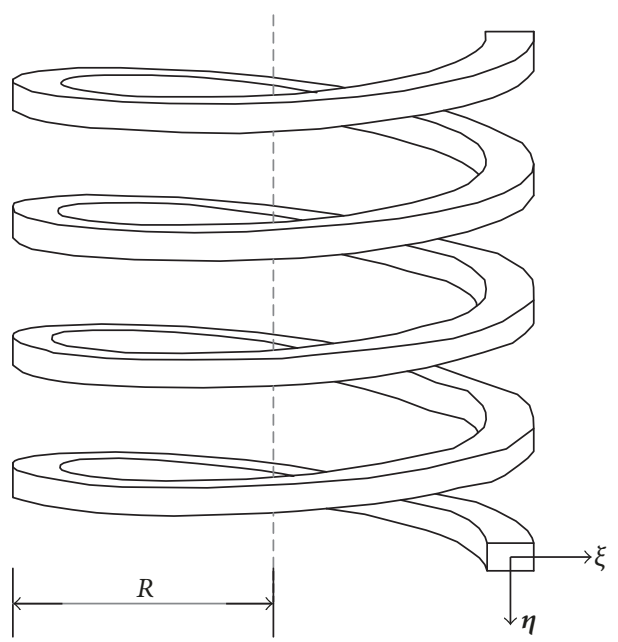

FIGURE 2: Cylindrical helical spring.

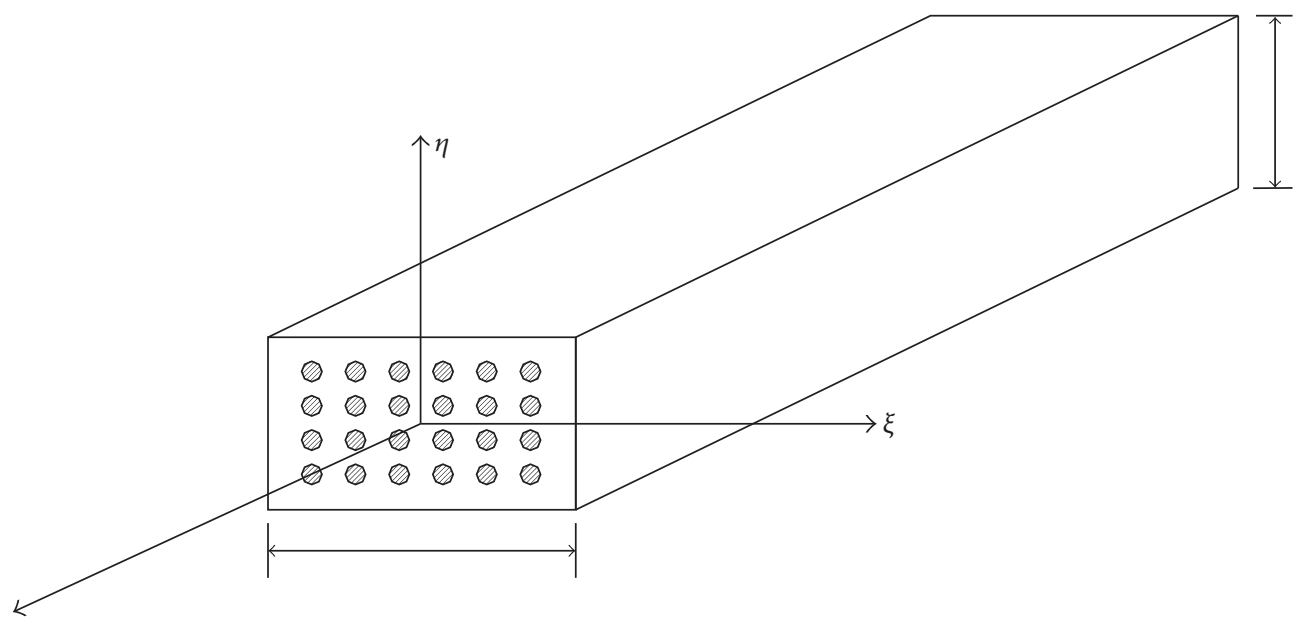

FIGURE 3: Unidirectional composite beam.

The differential equations of motion for cylindrical helical springs with rectangular cross section made of unidirectional composite materials are described as follows:

$$
\begin{aligned}
& \frac{\partial u_{s}(s, t)}{\partial s}=k_{\eta} u_{\xi}(s, t)+\left[\frac{k_{s} Q_{11} D_{1}\left(D_{10} D_{3} I_{2}+D_{2} D_{9} I_{3}-D_{12} I_{3} I_{2}\right)}{A_{11}\left(D_{3}^{2} I_{2}+D_{2}^{2} I_{3}-D_{11} I_{2} I_{3}\right)}-\frac{k_{s} Q_{11} D_{4}}{A_{11}}\right] \alpha(s, t)+\left[\frac{1}{A_{11}}\right] Q_{s}(s, t) \\
& \left.-\frac{Q_{11} D_{1}^{2} I_{2} I_{3}}{A_{11}\left(A_{11} D_{3}^{2} I_{2}+A_{11} D_{2}^{2} I_{3}-A_{11} D_{11} I_{2} I_{3}+Q_{11} D_{1}^{2} I_{2} I_{3}\right)}\right] \\
& -\left(\frac{D_{1} D_{2} I_{3}}{A_{11} D_{3}^{2} I_{2}+A_{11} D_{2}^{2} I_{3}-A_{11} D_{11} I_{2} I_{3}+Q_{11} D_{1}^{2} I_{2} I_{3}}\right) M_{\xi}(s, t)+\left(\frac{D_{1} D_{3} I_{2}}{A_{11} D_{3}^{2} I_{2}+A_{11} D_{2}^{2} I_{3}-A_{11} D_{11} I_{2} I_{3}+Q_{11} D_{1}^{2} I_{2} I_{3}}\right) \\
& \cdot M_{\eta}(s, t)+\left(\frac{D_{1} I_{2} I_{3}}{A_{11} D_{3}^{2} I_{2}+A_{11} D_{2}^{2} I_{3}-A_{11} D_{11} I_{2} I_{3}+Q_{11} D_{1}^{2} I_{2} I_{3}}\right) T(s, t),
\end{aligned}
$$




$$
\begin{aligned}
& \frac{\partial u_{\xi}(s, t)}{\partial s}=-k_{\eta} u_{s}(s, t)+k_{s} u_{\eta}(s, t)+\varphi_{\eta}(s, t)-\frac{D_{5} Q_{66}+k_{\eta} D_{1} Q_{66}}{A_{66} G_{\xi}} \alpha(s, t)+\frac{1}{A_{66} G_{\xi}} Q_{\xi}(s, t), \\
& \frac{\partial u_{\eta}(s, t)}{\partial s}=-k_{s} u_{\xi}(s, t)-\varphi_{\xi}(s, t)-\frac{D_{6} Q_{55}}{A_{55} G_{\eta}} \alpha(s, t)+\frac{1}{A_{55} G_{\eta}} Q_{\eta}(s, t), \\
& \frac{\partial \varphi_{s}(s, t)}{\partial s}=k_{\eta} \varphi_{\xi}(s, t)+\frac{-D_{7} Q_{55}+D_{8} Q_{66}+k_{\eta} D_{2} Q_{66}}{I_{3} Q_{55}+I_{2} Q_{66}} \alpha(s, t)+\frac{1}{I_{3} Q_{55}+I_{2} Q_{66}} M_{s}(s, t) \text {, } \\
& \frac{\partial \varphi_{\xi}(s, t)}{\partial s}=-k_{\eta} \varphi_{s}+k_{s} \varphi_{\eta}-\left(\frac{D_{1} D_{2} I_{3}}{A_{11}\left(D_{3}^{2} I_{2}+D_{2}^{2} I_{3}-D_{11} I_{2} I_{3}\right)+Q_{11} D_{1}^{2} I_{2} I_{3}}\right) Q_{s}(s, t) \\
& +\frac{k_{s}\left[A_{11}\left(D_{10} D_{3} D_{2}-D_{3}^{2} D_{9}-D_{2} D_{12} I_{3}+D_{11} D_{9} I_{3}\right)+Q_{11} I_{3} D_{1}\left(D_{2} D_{4}-D_{1} D_{9}\right)\right]}{A_{11}\left(D_{3}^{2} I_{2}+D_{2}^{2} I_{3}-D_{11} I_{2} I_{3}\right)+Q_{11} D_{1}^{2} I_{2} I_{3}} \alpha(s, t)+\left[\frac{1}{I_{2} Q_{11}}\right. \\
& \left.-\frac{A_{11} D_{2}^{2} I_{3}}{Q_{11} I_{2}\left[A_{11}\left(D_{3}^{2} I_{2}+D_{2}^{2} I_{3}-D_{11} I_{2} I_{3}\right)+Q_{11} D_{1}^{2} I_{2} I_{3}\right]}\right] M_{\xi}(s, t)+\frac{A_{11} D_{2} D_{3}}{Q_{11}\left[A_{11}\left(D_{3}^{2} I_{2}+D_{2}^{2} I_{3}-D_{11} I_{2} I_{3}\right)+Q_{11} D_{1}^{2} I_{2} I_{3}\right]} \\
& \cdot M_{\eta}(s, t)+\frac{A_{11} D_{2} I_{3}}{Q_{11}\left[A_{11}\left(D_{3}^{2} I_{2}+D_{2}^{2} I_{3}-D_{11} I_{2} I_{3}\right)+Q_{11} D_{1}^{2} I_{2} I_{3}\right]} T(s, t), \\
& \frac{\partial \varphi_{\eta}(s, t)}{\partial s}=-k_{s} \varphi_{\xi}(s, t)+\left(\frac{D_{1} D_{3} I_{2}}{A_{11}\left(D_{3}^{2} I_{2}+D_{2}^{2} I_{3}-D_{11} I_{2} I_{3}\right)+Q_{11} D_{1}^{2} I_{2} I_{3}}\right) Q_{s}(s, t) \\
& +\frac{k_{s}\left[A_{11}\left(D_{10} D_{2}^{2}-D_{3} D_{2} D_{9}+D_{3} D_{12} I_{2}-D_{10} D_{11} I_{2}\right)+Q_{11} I_{2} D_{1}\left(D_{1} D_{10}-D_{3} D_{4}\right)\right]}{A_{11}\left(D_{3}^{2} I_{2}+D_{2}^{2} I_{3}-D_{11} I_{2} I_{3}\right)+Q_{11} D_{1}^{2} I_{2} I_{3}} \alpha(s, t) \\
& +\frac{A_{11} D_{2} D_{3}}{Q_{11}\left[A_{11}\left(D_{3}^{2} I_{2}+D_{2}^{2} I_{3}-D_{11} I_{2} I_{3}\right)+Q_{11} D_{1}^{2} I_{2} I_{3}\right]} M_{\xi}(s, t)+\left[\frac{1}{I_{3} Q_{11}}\right. \\
& \left.-\frac{A_{11} D_{3}^{2} I_{2}}{Q_{11} I_{3}\left[A_{11}\left(D_{3}^{2} I_{2}+D_{2}^{2} I_{3}-D_{11} I_{2} I_{3}\right)+Q_{11} D_{1}^{2} I_{2} I_{3}\right]}\right] M_{\eta}(s, t)-\frac{A_{11} D_{3} I_{2}}{Q_{11}\left[A_{11}\left(D_{3}^{2} I_{2}+D_{2}^{2} I_{3}-D_{11} I_{2} I_{3}\right)+Q_{11} D_{1}^{2} I_{2} I_{3}\right]} \\
& \text { - } T(s, t) \text {, } \\
& \frac{\partial \alpha(s, t)}{\partial s}=-\frac{k_{s}\left[A_{11}\left(D_{10} D_{3} I_{2}+I_{3} D_{2} D_{9}-I_{3} D_{12} I_{2}\right)+Q_{11} D_{1} D_{4} I_{2} I_{3}\right]}{A_{11}\left(D_{3}^{2} I_{2}+D_{2}^{2} I_{3}-D_{11} I_{2} I_{3}\right)+Q_{11} D_{1}^{2} I_{2} I_{3}} \alpha(s, t) \\
& +\frac{D_{1} I_{3} I_{2}}{A_{11}\left(D_{3}^{2} I_{2}+D_{2}^{2} I_{3}-D_{11} I_{2} I_{3}\right)+Q_{11} D_{1}^{2} I_{2} I_{3}} Q_{s}(s, t)+\frac{A_{11} D_{2} I_{3}}{Q_{11}\left[A_{11}\left(D_{3}^{2} I_{2}+D_{2}^{2} I_{3}-D_{11} I_{2} I_{3}\right)+Q_{11} D_{1}^{2} I_{2} I_{3}\right]} M_{\xi}(s, t) \\
& -\frac{A_{11} D_{3} I_{2}}{Q_{11}\left[A_{11}\left(D_{3}^{2} I_{2}+D_{2}^{2} I_{3}-D_{11} I_{2} I_{3}\right)+Q_{11} D_{1}^{2} I_{2} I_{3}\right]} M_{\eta}(s, t)-\frac{A_{11} I_{3} I_{2}}{Q_{11}\left[A_{11}\left(D_{3}^{2} I_{2}+D_{2}^{2} I_{3}-D_{11} I_{2} I_{3}\right)+Q_{11} D_{1}^{2} I_{2} I_{3}\right]} \\
& \text { - } T(s, t) \text {, } \\
& \frac{\partial Q_{s}(s, t)}{\partial s}=k_{\eta} Q_{\xi}(s, t)+\rho A \frac{\partial^{2} u_{s}(s, t)}{\partial t^{2}}+\rho D_{1} \frac{\partial^{2} \alpha(s, t)}{\partial t^{2}} \\
& \frac{\partial Q_{\xi}(s, t)}{\partial s}=-k_{\eta} Q_{s}(s, t)+k_{s} Q_{\eta}(s, t)+\rho A \frac{\partial^{2} u_{\xi}(s, t)}{\partial t^{2}} \\
& \frac{\partial Q_{\eta}(s, t)}{\partial s}=-k_{s} Q_{\xi}(s, t)+\rho A \frac{\partial^{2} u_{\eta}(s, t)}{\partial t^{2}}, \\
& \frac{\partial M_{s}(s, t)}{\partial s}=k_{\eta} M_{\xi}(s, t)+\rho I_{1} \frac{\partial^{2} \varphi_{s}(s, t)}{\partial t^{2}},
\end{aligned}
$$




$$
\begin{aligned}
& \frac{\partial M_{\xi}(s, t)}{\partial s}=Q_{\eta}(s, t)-k_{\eta} M_{s}(s, t)+k_{s} M_{\eta}(s, t)+\rho I_{2} \frac{\partial^{2} \varphi_{\xi}(s, t)}{\partial t^{2}}+\rho D_{2} \frac{\partial^{2} \alpha(s, t)}{\partial t^{2}}, \\
& \frac{\partial M_{\eta}(s, t)}{\partial s}=-Q_{\xi}-k_{s} M_{\xi}+\rho I_{3} \frac{\partial^{2} \varphi_{\eta}(s, t)}{\partial t^{2}}-\rho D_{3} \frac{\partial^{2} \alpha(s, t)}{\partial t^{2}}, \\
& \frac{\partial T(s, t)}{\partial s}=\rho D_{11} \ddot{\alpha}+\rho D_{1} \ddot{u}_{s}+\rho D_{2} \ddot{\varphi}_{\xi}-\rho D_{3} \ddot{\varphi}_{\eta}+\left\{D_{16} Q_{55}+D_{14} Q_{66}+\left(2 D_{15}+D_{11} k_{\eta}\right) k_{\eta} Q_{66}+D_{13} k_{s}^{2} Q_{11}\right. \\
& -\frac{\left(D_{7} Q_{55}-\left(D_{8}+D_{2} k_{\eta}\right) Q_{66}\right)^{2}}{I_{3} Q_{55}+I_{2} Q_{66}}+\frac{D_{3}^{2} D_{10}^{2} I_{2} k_{s}^{2} Q_{11} A_{11}}{I_{3}\left(A_{11}\left(D_{3}^{2} I_{2}+D_{2}^{2} I_{3}-D_{11} I_{2} I_{3}\right)+Q_{11} D_{1}^{2} I_{2} I_{3}\right)} \\
& -\frac{2 k_{s}^{2} Q_{11} A_{11}\left(D_{12} D_{10} D_{3} I_{2}+D_{9} D_{12} D_{2} I_{3}\right)}{A_{11}\left(D_{3}^{2} I_{2}+D_{2}^{2} I_{3}-D_{11} I_{2} I_{3}\right)+Q_{11} D_{1}^{2} I_{2} I_{3}}+\frac{2 D_{9} D_{10} D_{3} D_{4} k_{s}^{2} Q_{11} A_{11}}{A_{11}\left(D_{3}^{2} I_{2}+D_{2}^{2} I_{3}-D_{11} I_{2} I_{3}\right)+Q_{11} D_{1}^{2} I_{2} I_{3}} \\
& +\frac{D_{12}^{2} I_{2} I_{3} k_{s}^{2} Q_{11} A_{11}}{A_{11}\left(D_{3}^{2} I_{2}+D_{2}^{2} I_{3}-D_{11} I_{2} I_{3}\right)+Q_{11} D_{1}^{2} I_{2} I_{3}}+\frac{2 k_{s}^{2} Q_{11}^{2} D_{1} D_{4}\left(D_{10} D_{3} I_{2}+D_{2} D_{9} I_{3}\right)}{A_{11}\left(D_{3}^{2} I_{2}+D_{2}^{2} I_{3}-D_{11} I_{2} I_{3}\right)+Q_{11} D_{1}^{2} I_{2} I_{3}} \\
& +\frac{D_{2}^{2} D_{9}^{2} I_{3} k_{s}^{2} Q_{11} A_{11}}{I_{2}\left(A_{11}\left(D_{3}^{2} I_{2}+D_{2}^{2} I_{3}-D_{11} I_{2} I_{3}\right)+Q_{11} D_{1}^{2} I_{2} I_{3}\right)}-\frac{k_{s}^{2} Q_{11} D_{9}^{2}}{I_{2}}-\frac{k_{s}^{2} Q_{11} D_{10}^{2}}{I_{3}}-\frac{k_{s}^{2} Q_{11}^{2} D_{4}^{2}}{A_{11}} \\
& \left.+\frac{D_{1}^{2} D_{4}^{2} I_{2} I_{3} k_{s}^{2} Q_{11}^{3}}{A_{11}\left(A_{11}\left(D_{3}^{2} I_{2}+D_{2}^{2} I_{3}-D_{11} I_{2} I_{3}\right)+Q_{11} D_{1}^{2} I_{2} I_{3}\right)}-\frac{D_{6}^{2} Q_{55}^{2}}{A_{55}}-\frac{\left(D_{5}+D_{1} k_{\eta}\right)^{2} Q_{66}^{2}}{A_{66}}\right\} \alpha(s, t) \\
& +\frac{k_{s} Q_{11}\left[D_{4}\left(D_{3}^{2} I_{2}+D_{2}^{2} I_{3}-D_{11} I_{2} I_{3}\right)-D_{1}\left(D_{10} D_{3} I_{2}+D_{2} D_{9} I_{3}-D_{12} I_{3} I_{2}\right)\right]}{A_{11}\left(D_{3}^{2} I_{2}+D_{2}^{2} I_{3}-D_{11} I_{2} I_{3}\right)+Q_{11} D_{1}^{2} I_{2} I_{3}} Q_{s}(s, t)+\frac{\left(D_{5}+D_{1} k_{\eta}\right) Q_{66}}{A_{66}} Q_{\xi}(s, t) \\
& +\frac{D_{6} Q_{55}}{A_{55}} Q_{\eta}(s, t)+\frac{D_{7} Q_{55}-\left(D_{8}+D_{2} k_{\eta}\right) Q_{66}}{I_{3} Q_{55}+I_{2} Q_{66}} M_{s}(s, t) \\
& -\frac{k_{s} A_{11}\left(D_{10} D_{2} D_{3}-D_{3}^{2} D_{9}-D_{12} D_{2} I_{3}+D_{11} D_{9} I_{3}\right)+k_{s} Q_{11} D_{1} I_{3}\left(D_{2} D_{4}-D_{1} D_{9}\right)}{A_{11}\left(D_{3}^{2} I_{2}+D_{2}^{2} I_{3}-D_{11} I_{2} I_{3}\right)+Q_{11} D_{1}^{2} I_{2} I_{3}} M_{\xi}(s, t) \\
& -\frac{k_{s} A_{11}\left(D_{9} D_{2} D_{3}+D_{2}^{2} D_{10}+D_{12} D_{3} I_{2}-D_{11} D_{10} I_{2}\right)+k_{s} Q_{11} D_{1} I_{2}\left(D_{1} D_{10}-D_{3} D_{4}\right)}{A_{11}\left(D_{3}^{2} I_{2}+D_{2}^{2} I_{3}-D_{11} I_{2} I_{3}\right)+Q_{11} D_{1}^{2} I_{2} I_{3}} M_{\eta}(s, t) \\
& +\frac{k_{s} A_{11}\left(D_{9} D_{2} I_{3}+D_{10} D_{3} I_{2}-D_{12} I_{2} I_{3}\right)+k_{s} Q_{11} D_{4} I_{2} I_{3}}{A_{11}\left(D_{3}^{2} I_{2}+D_{2}^{2} I_{3}-D_{11} I_{2} I_{3}\right)+Q_{11} D_{1}^{2} I_{2} I_{3}} T(s, t),
\end{aligned}
$$

where

$$
\begin{aligned}
A_{11} & =\iint_{A} Q_{11} d \xi d \eta, \\
A_{55} & =\iint_{A} Q_{55} d \xi d \eta, \\
A_{66} & =\iint_{A} Q_{66} d \xi d \eta, \\
D_{1} & =\iint_{A} \varphi d \xi d \eta, \\
D_{2} & =\iint_{A} \varphi \eta d \xi d \eta,
\end{aligned}
$$

$$
\begin{aligned}
& D_{3}=\iint_{A} \varphi \xi d \xi d \eta \\
& D_{4}=\iint_{A}\left[\left(\frac{\partial \varphi}{\partial \xi}\right) \eta-\left(\frac{\partial \varphi}{\partial \eta}\right) \xi\right] d \xi d \eta \\
& D_{5}=\iint_{A} \frac{\partial \varphi}{\partial \xi} d \xi d \eta \\
& D_{6}=\iint_{A} \frac{\partial \varphi}{\partial \eta} d \xi d \eta \\
& D_{7}=\iint_{A} \xi \frac{\partial \varphi}{\partial \eta} d \xi d \eta
\end{aligned}
$$




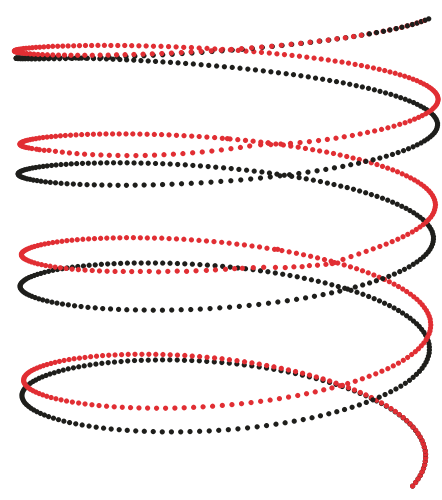

(1)

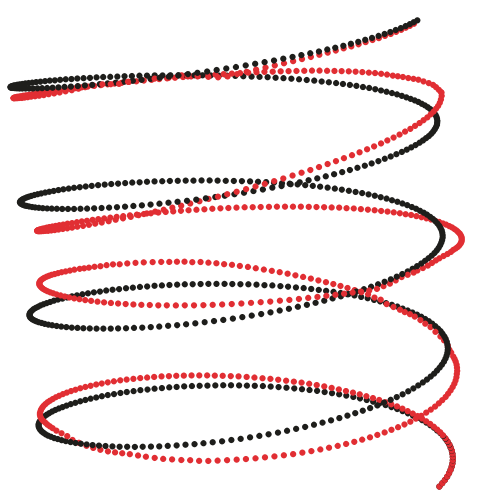

(2)

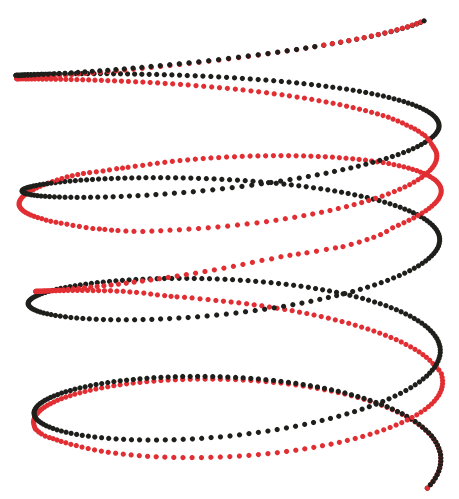

(3)

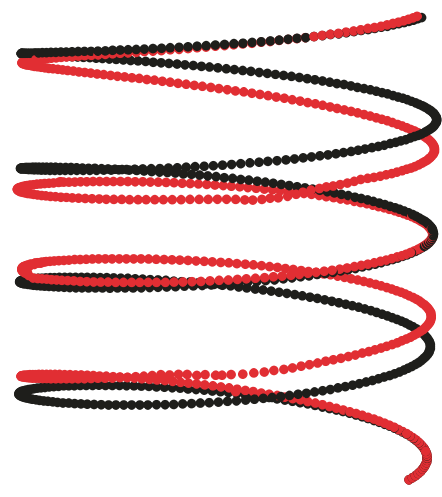

(4)

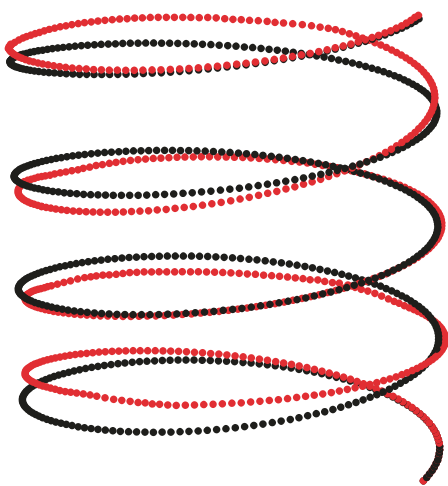

(5)

Figure 4: First five mode shapes of Beam 1: (1) first mode (523.25 Hz); (2) second mode (897.71 Hz); (3) third mode (914.36 Hz); (4) fourth mode $(973.88 \mathrm{~Hz})$; (5) fifth mode $(1118.41 \mathrm{~Hz})$.

$$
\begin{aligned}
D_{8} & =\iint_{A} \eta \frac{\partial \varphi}{\partial \xi} d \xi d \eta, \\
D_{12} & =\iint_{A} \varphi\left[\left(\frac{\partial \varphi}{\partial \xi}\right) \eta-\left(\frac{\partial \varphi}{\partial \eta}\right) \xi\right] d \xi d \eta \\
D_{9} & =\iint_{A} \eta\left[\left(\frac{\partial \varphi}{\partial \xi}\right) \eta-\left(\frac{\partial \varphi}{\partial \eta}\right) \xi\right] d \xi d \eta, \\
D_{10} & =\iint_{A} \xi\left[\left(\frac{\partial \varphi}{\partial \xi}\right) \eta-\left(\frac{\partial \varphi}{\partial \eta}\right) \xi\right] d \xi d \eta, \\
D_{11} & =\iint_{A} \varphi^{2} d \xi d \eta \\
D_{13} & =\iint_{A}\left[\left(\frac{\partial \varphi}{\partial \xi}\right) \eta-\left(\frac{\partial \varphi}{\partial \eta}\right) \xi\right]^{2} d \xi d \eta, \\
D_{14} & =\iint_{A}\left(\frac{\partial \varphi}{\partial \xi}\right)^{2} d \xi d \eta, \\
D_{15} & =\iint_{A} \varphi\left(\frac{\partial \varphi}{\partial \xi}\right) d \xi d \eta \\
D_{16} & =\iint_{A}\left(\frac{\partial \varphi}{\partial \eta}\right)^{2} d \xi d \eta .
\end{aligned}
$$

5.2. Effects of Material on the Natural Frequencies with Warping. The geometric parameters and finite element meshes of the two beams are exactly the same: the cylinder diameter $R=5 \mathrm{~mm}$, the helix pitch angle $\bar{\alpha}=5^{\circ}$, the number of active turns $n=4$, and the rectangular cross section of $2 a=1 \mathrm{~mm}$ in the $\xi$-direction and $2 b=0.4 \mathrm{~mm}$ in the $\eta$-direction. The only difference between them is the material properties. Beam 1 is made of isotropic materials (the density is $\rho=7900 \mathrm{~kg} / \mathrm{m}^{3}$, the modulus of elasticity is $E=206 \mathrm{GPa}$, and Poisson's ratio is $\mu=0.3$ ), while Beam 2 is made of unidirectional composite materials (the modulus of elasticity in the three main directions is $E_{1}=181 \mathrm{Gpa}, E_{2}=E_{3}=10.3 \mathrm{Gpa}$, the modulus of shearing of the three planes is $G_{23}=3.433 \mathrm{Gpa}$, $G_{12}=G_{13}=7.17 \mathrm{Gpa}$, the main Poisson's ratio is $\mu_{23}=0.5$, $\mu_{12}=\mu_{13}=0.28$, and the density is $\left.\rho=1600 \mathrm{~kg} / \mathrm{m}^{3}\right)$. We partitioned Beam 1 (Beam 2) into 720 Solid45 (Solid46) elements using ANSYS for analysis. The total number of nodal points is 1452 . The first five natural frequencies of Beam 1 and Beam 2 are listed in Tables 1 and 2. The first five normal mode shapes of beams and the generalized warping coordinate are demonstrated in Figures 4-7, respectively. 


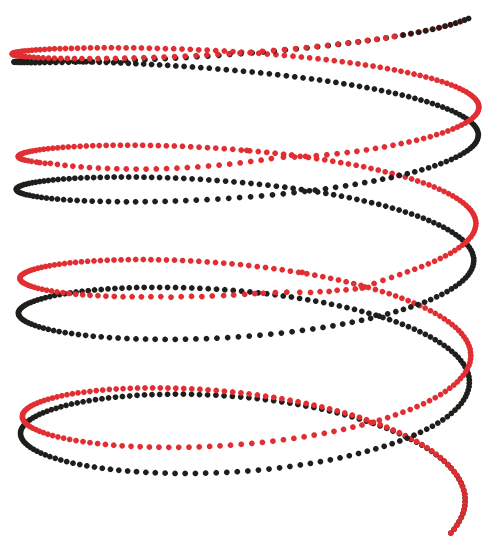

(a)

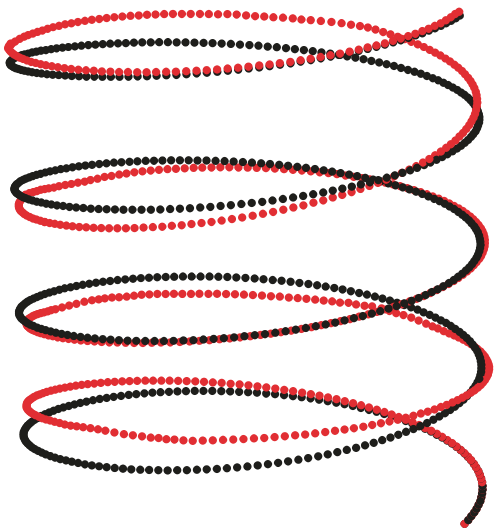

(c)

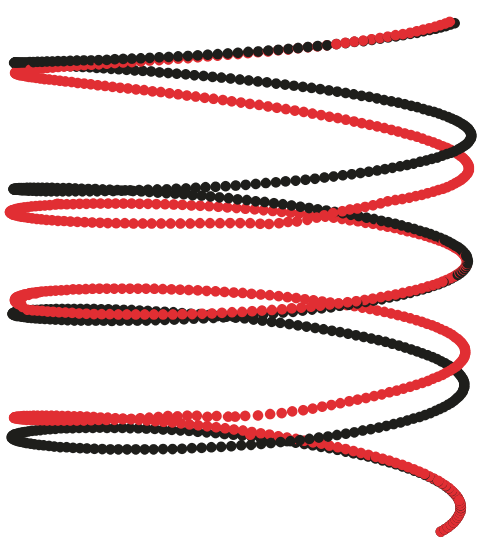

(b)

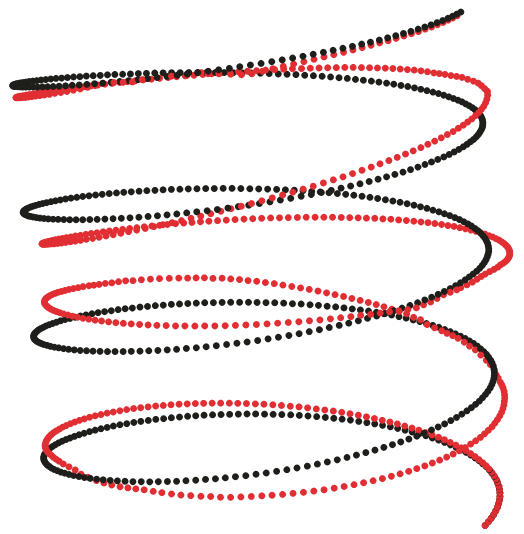

(d)

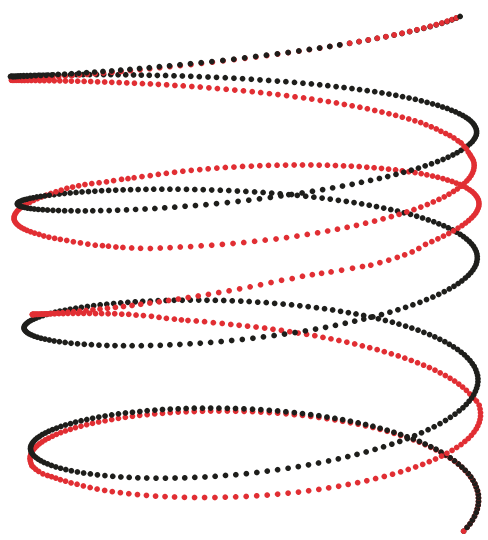

(e)

Figure 5: First five mode shapes of Beam 2 (made of unidirectional composite material): (a) first mode (358.21 Hz); (b) second mode $(687.63 \mathrm{~Hz})$; (c) third mode $(805.74 \mathrm{~Hz})$; (d) fourth mode $(903.08 \mathrm{~Hz})$; (e) fifth mode $(958.56 \mathrm{~Hz})$.

\section{Conclusion}

The differential equations of motion for elastic space beams with noncircular cross sections and anisotropic materials have been derived systematically based on naturally curved and twisted beam theory. In the equations, the warping deformation of the cross section, the initial twist of the cross section, the shear and axial deformations, and the rotary inertia are studied theoretically.

The results show that the effect of warping deformations on the natural frequencies of the beam is very significant under certain geometric and boundary conditions. The errors can be excessively large when the warping effect is disregarded. The influence of warping on the natural frequency is enhanced with the increase in the anisotropy of materials. 
TABLE 1: The first five frequencies of Beam 1 (made of isotropic material).

\begin{tabular}{|c|c|c|c|c|c|}
\hline \multirow{2}{*}{ Mode number $(\mathrm{Hz})$} & \multirow{2}{*}{ FEM } & \multicolumn{4}{|c|}{ Present study (PS) } \\
\hline & & Warping ignored & Errors (\%) & Warping included & Errors (\%) \\
\hline 1 & 521.34 & 745.84 & 43.06 & 523.25 & 0.37 \\
\hline 2 & 899.54 & 1009.30 & 12.20 & 897.71 & 0.20 \\
\hline 3 & 915.97 & 1044.17 & 13.99 & 914.36 & 0.17 \\
\hline 4 & 970.21 & 1122.22 & 15.67 & 973.88 & 0.38 \\
\hline 5 & 1129.5 & 1338.65 & 18.52 & 1118.41 & 0.98 \\
\hline
\end{tabular}

TABLE 2: The first five frequencies of Beam 2 (made of unidirectional composite material).

\begin{tabular}{|c|c|c|c|c|c|}
\hline \multirow{2}{*}{ Mode number $(\mathrm{Hz})$} & \multirow{2}{*}{ FEM } & \multicolumn{4}{|c|}{ Present study (PS) } \\
\hline & & Warping ignored & Errors (\%) & Warping included & Errors (\%) \\
\hline 1 & 351.54 & 512.80 & 45.87 & 358.21 & 1.89 \\
\hline 2 & 675.19 & 981.32 & 45.34 & 687.63 & 1.84 \\
\hline 3 & 787.86 & 1117.87 & 41.89 & 805.74 & 2.27 \\
\hline 4 & 880.95 & 1200.09 & 36.23 & 903.08 & 2.51 \\
\hline 5 & 940.96 & 1357.22 & 44.24 & 958.56 & 1.87 \\
\hline
\end{tabular}

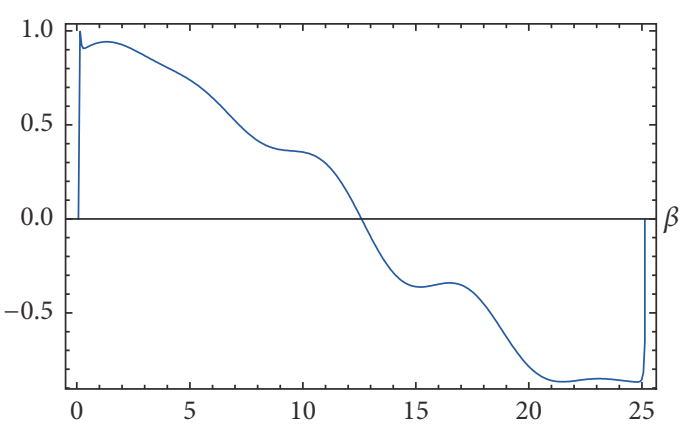

(a) First mode $(523.25 \mathrm{~Hz})$

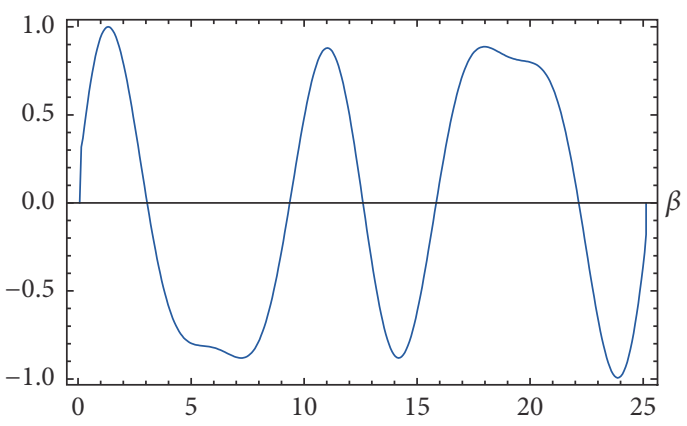

(c) Third mode $(914.36 \mathrm{~Hz})$

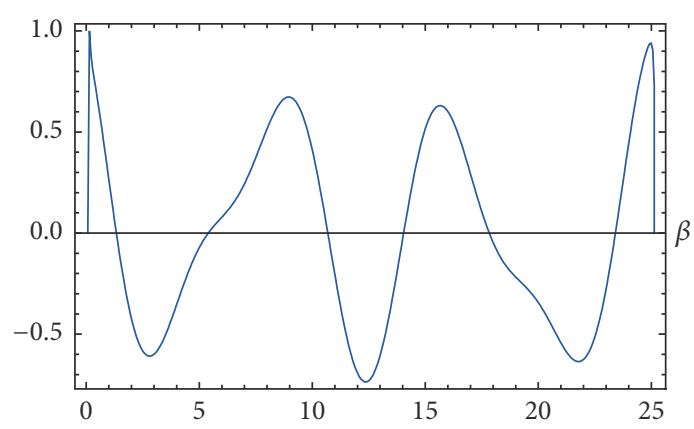

(b) Second mode $(897.71 \mathrm{~Hz})$

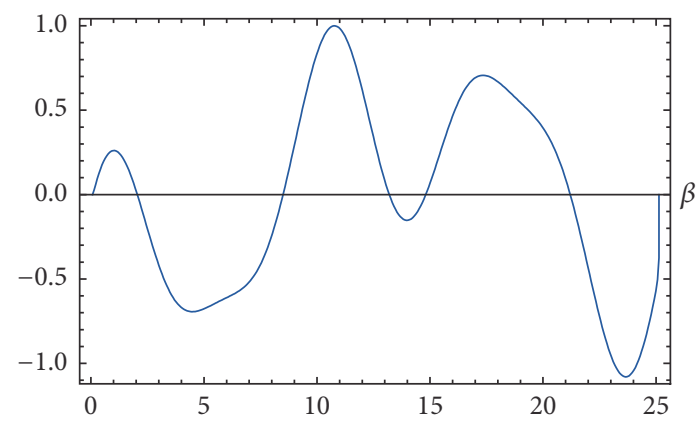

(d) Fourth mode $(973.88 \mathrm{~Hz})$

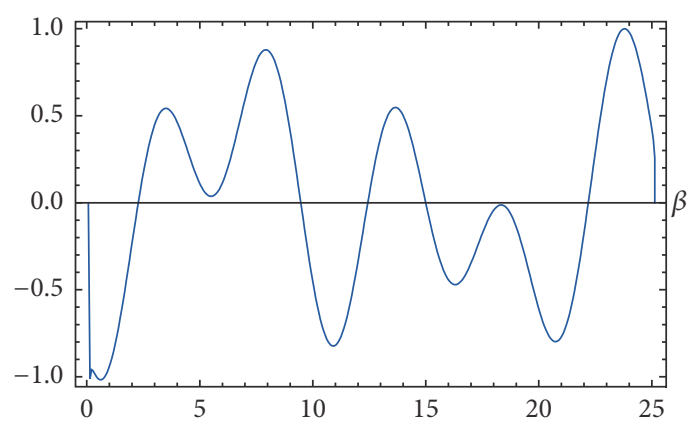

(e) Fifth mode $(1118.41 \mathrm{~Hz})$

FIgURE 6: First five normal mode shapes of the generalized warping coordinate of Beam 1. 


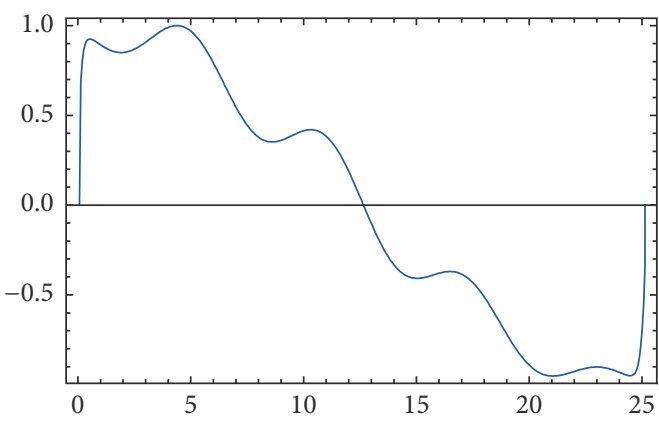

(a) First mode $(358.21 \mathrm{~Hz})$

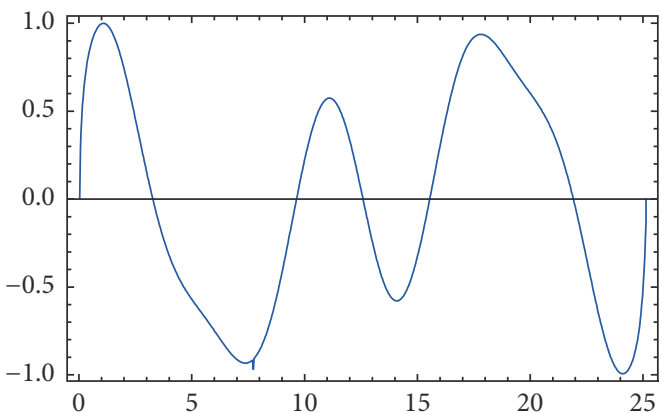

(c) Third mode $(805.74 \mathrm{~Hz})$

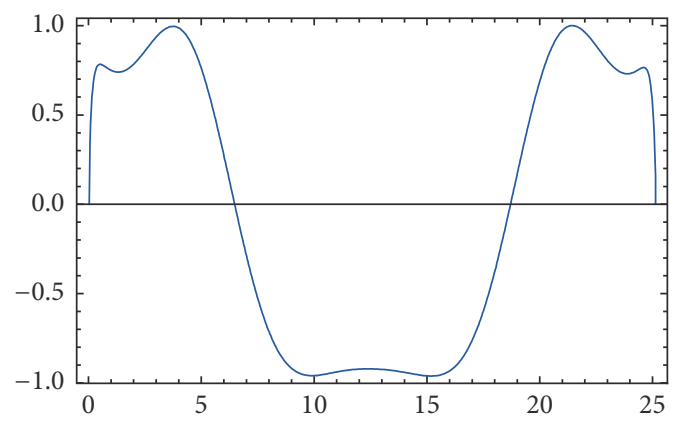

(b) Second mode $(687.63 \mathrm{~Hz})$

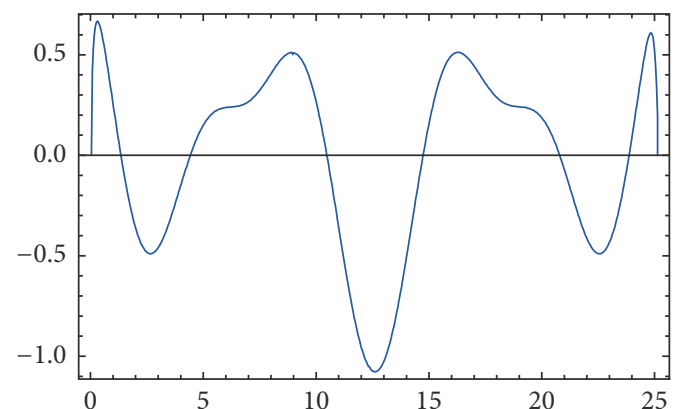

(d) Fourth mode $(903.08 \mathrm{~Hz})$

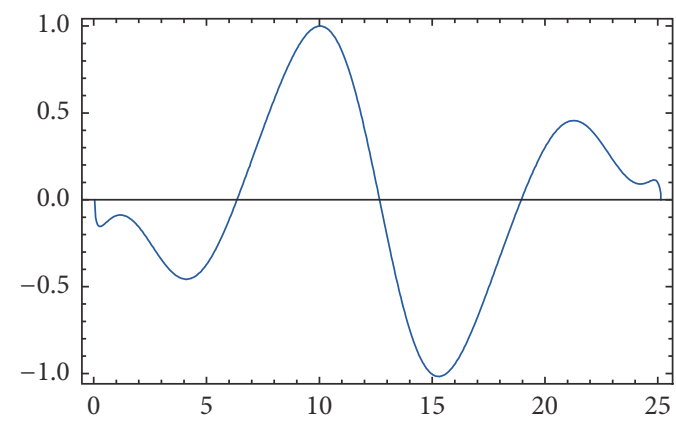

(e) Fifth mode $(958.56 \mathrm{~Hz})$

FIGURE 7: First five normal mode shapes of the generalized warping coordinate of Beam 2.

\section{Conflicts of Interest}

The authors declare that they have no conflicts of interest.

\section{Acknowledgments}

This work is partially supported by National Natural Science Foundation of China (11402090, 51779095, and 11502081).

\section{References}

[1] K. Chandrashekhara, K. Krishnamurthy, and S. Roy, "Free vibration of composite beams including rotary inertia and shear deformation," Composite Structures, vol. 14, no. 4, pp. 269-279, 1990.

[2] M. P. Singh and A. S. Abdelnaser, "Random response of symmetric cross-ply composite beams with arbitrary boundary conditions," AIAA Journal, vol. 30, no. 4, pp. 1081-1088, 1992.
[3] J. Li, H. Hongxing, and S. Rongying, "Dynamic finite element method for generally laminated composite beams," International Journal of Mechanical Sciences, vol. 50, no. 3, pp. 466-480, 2008.

[4] R. S. Ramalingeswara and N. Ganesan, "Dynamic response of non-uniform composite beams," Journal of Sound and Vibration, vol. 200, no. 5, pp. 563-577, 1997.

[5] J. A. Zapfe and G. A. Lesieutre, "Vibration analysis of laminated beams using an iterative smeared laminate model," Journal of Sound and Vibration, vol. 199, no. 2, pp. 275-284, 1997.

[6] V. Yildirim, "Rotary inertia, axial and shear deformation effects on the in-plane natural frequencies of symmetric cross-ply laminated circular arches," Journal of Sound and Vibration, vol. 224, no. 4, pp. 575-589, 1999.

[7] Y. P. Tseng, C. S. Huang, and M. S. Kao, "In-plane vibration of laminated curved beams with variable curvature by dynamic stiffness analysis," Composite Structures, vol. 50, no. 2, pp. 103$114,2000$. 
[8] H. Matsunaga, "In-plane vibration and stability of shallow circular arches subjected to axial forces," International Journal of Solids and Structures, vol. 33, no. 4, pp. 469-482, 1996.

[9] H. Matsunaga, "Effects of higher-order deformations on inplane vibration and stability of thick circular rings," Acta Mechanica, vol. 124, no. 1-4, pp. 47-61, 1997.

[10] B. O. Baba and S. Thoppul, "Experimental evaluation of the vibration behavior of flat and curved sandwich composite beams with face/core debond," Composite Structures, vol. 91, no. 1, pp. 110-119, 2009.

[11] V. Yildirim, "Governing equations of initially twisted elastic space rods made of laminated composite materials," International Journal of Engineering Science, vol. 37, no. 8, pp. 1007-1035, 1999.

[12] J.-S. Kim, M. Cho, and E. C. Smith, "An asymptotic analysis of composite beams with kinematically corrected end effects," International Journal of Solids and Structures, vol. 45, no. 7-8, pp. 1954-1977, 2008.

[13] E. J. Sapountzakis and V. G. Mokos, "Dynamic analysis of 3$\mathrm{D}$ beam elements including warping and shear deformation effects," International Journal of Solids and Structures, vol. 43, no. 22-23, pp. 6707-6726, 2006.

[14] W. Yu, D. H. Hodges, V. Volovoi, and C. E. S. Cesnik, "On Timoshenko-like modeling of initially curved and twisted composite beams," International Journal of Solids and Structures, vol. 39, no. 19, pp. 5101-5121, 2002.

[15] E. Ghafari and J. Rezaeepazhand, "Vibration analysis of rotating composite beams using polynomial based dimensional reduction method," International Journal of Mechanical Sciences, vol. 115-116, pp. 93-104, 2016.

[16] E. J. Sapountzakis and I. N. Tsiptsis, "B-splines in the Analog Equation Method for the generalized beam analysis including warping effects," Computers \& Structures, vol. 180, pp. 60-73, 2017.

[17] I. N. Tsiptsis and E. J. Sapountzakis, "Generalized warping and distortional analysis of curved beams with isogeometric methods," Computers \& Structures, vol. 191, pp. 33-50, 2017.

[18] E. J. Sapountzakis and I. N. Tsiptsis, "Generalized vibration analysis of beams including warping effects by isogeometric methods," Journal of Vibration \& Control, Article ID $107754631668567,2017$.

[19] I. N. Tsiptsis and E. J. Sapountzakis, "Isogeometric analysis for the dynamic problem of curved structures including warping effects," Mechanics Based Design of Structures and Machines, pp. 1-19, 2017.

[20] A.-M. Yu and Y. Hao, "Warping effect in free vibration analysis of unidirectional composite non-cylindrical helical springs," Meccanica, vol. 48, no. 10, pp. 2453-2465, 2013.

[21] A. M. Yu and Y. Hao, "Effect of warping on natural frequencies of symmetrical cross-ply laminated composite non-cylindrical helical springs," International Journal of Mechanical Sciences, vol. 74, pp. 65-72, 2013.

[22] A. M. Yu and Y. Hao, "Free vibration analysis of cylindrical helical springs with noncircular cross-sections," Journal of Sound and Vibration, vol. 330, no. 11, pp. 2628-2639, 2011.

[23] R. Q. Xu, J. S. He, and W. Q. Chen, "Saint-Venant torsion of orthotropic bars with inhomogeneous rectangular cross section," Composite Structures, vol. 92, no. 6, pp. 1449-1457, 2010.

[24] Y. Hao and J. Guan, "Parametric study on the influence of warping deformation upon natural frequencies of die springs," Shock and Vibration, vol. 2016, Article ID 6231676, 2016. 


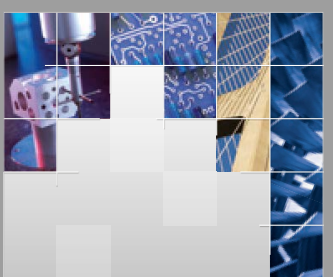

\section{Enfincering}
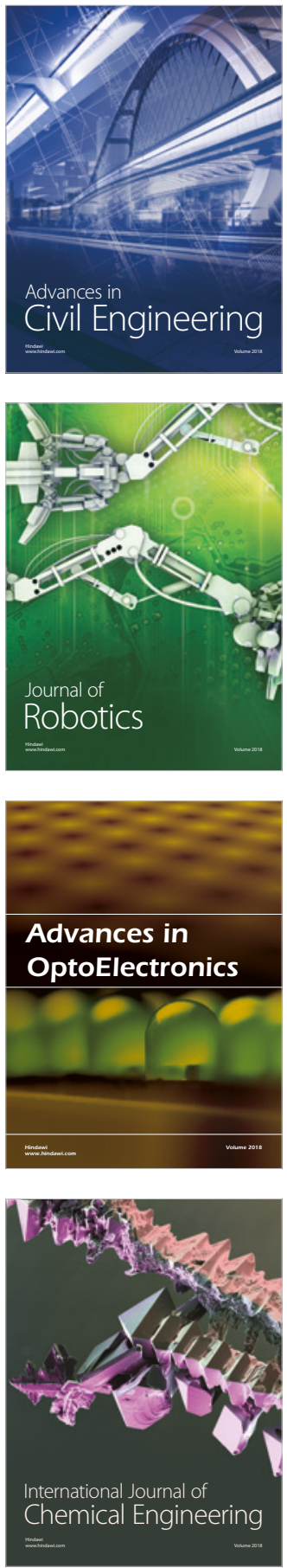

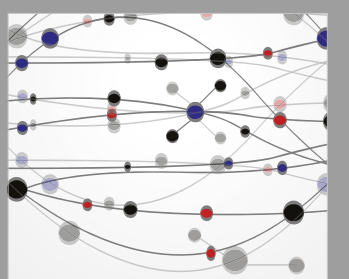

\section{Rotating \\ Machinery}

The Scientific World Journal

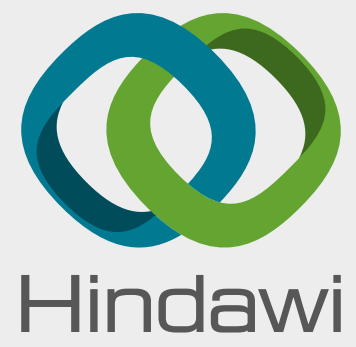

Submit your manuscripts at

www.hindawi.com
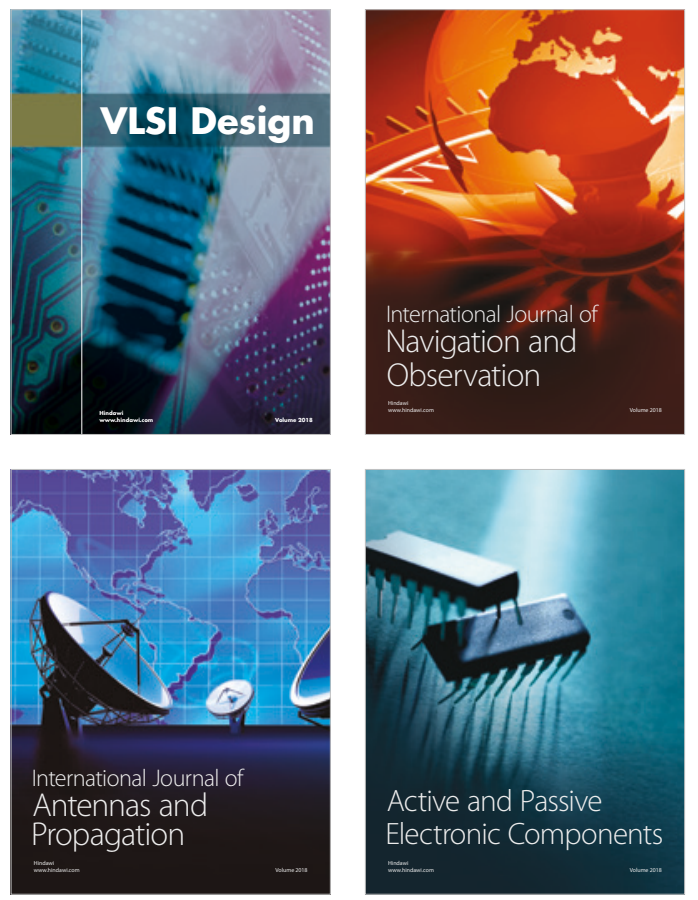
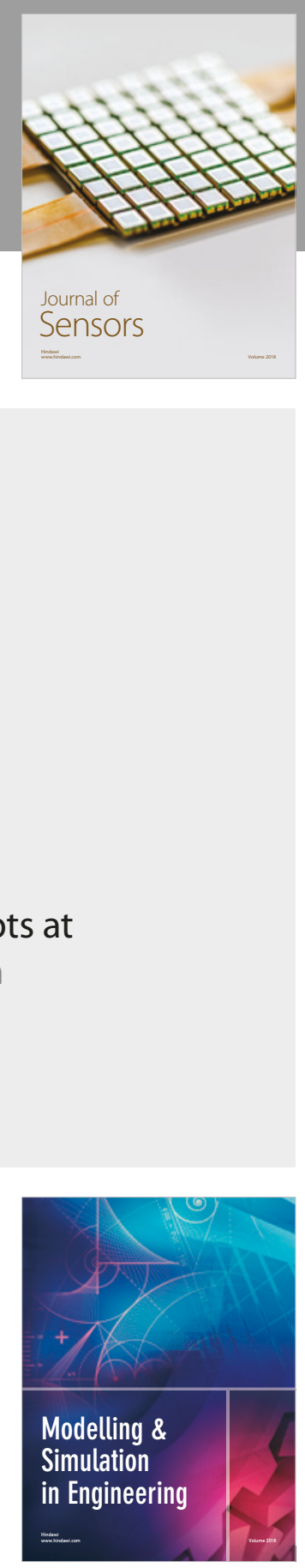

\section{Advances \\ Multimedia}
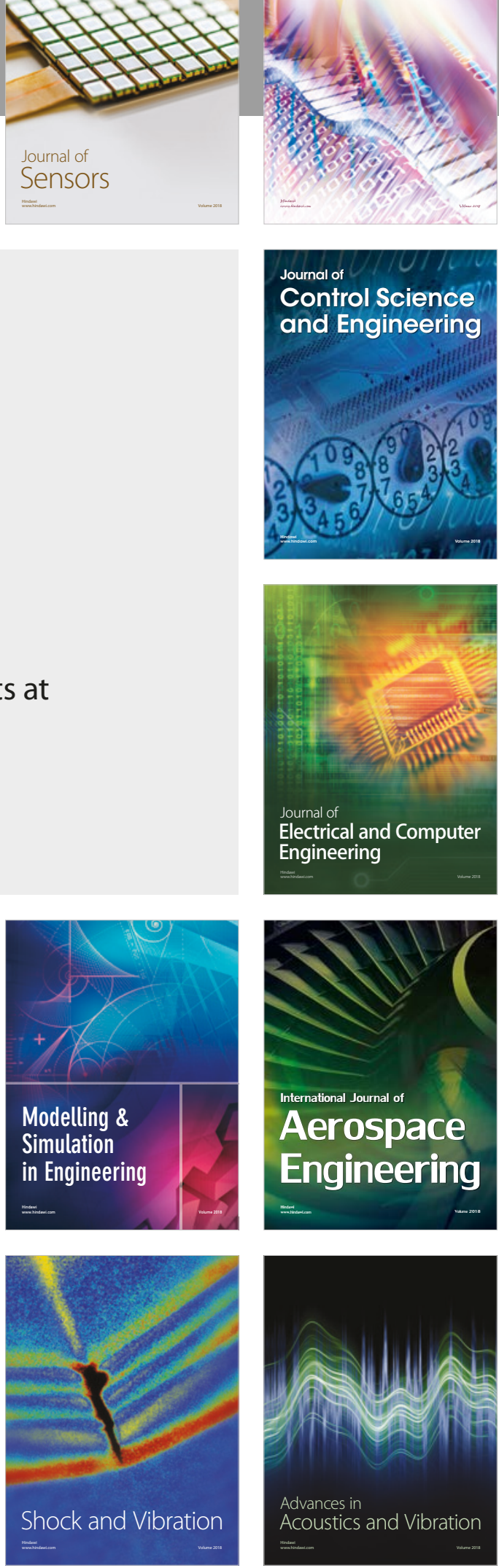